\title{
Reducing returns to theatre for neck of femur fracture patients
}

\author{
Selina Graham, Mark Dahill, Derek Robinson
}

To cite: Graham S, Dahill M, Robinson D. Reducing returns to theatre for neck of femur fracture patients. BMJ Quality Improvement Reports 2017;6:u215756.w6261. doi:10.1136/bmjquality. u215756.w6261

\section{CrossMark}

Royal United Hospitals Bath, United Kingdom

\section{ABSTRACT}

The Royal United Hospital, Bath, admits approximately 550 patients with neck of femur fractures per year. The risks from returning to theatre for this patient group are often life-threatening. Post-operative wound ooze was noted to cause a significant rate of return to theatre, with increased lengths of stay and patient morbidity.

A wound closure protocol was agreed by the consultant body. This information was disseminated by email and teaching sessions to all members of the multidisciplinary team, including surgeons, theatre staff and ortho-geriatricians. The plan-do-study-act model for improvement was used to reduce rates of returns to theatre for wound ooze. Interventions included cyclical teaching during each trainee rotation, updated inductions, posters, email reminders and scrub team involvement to open the protocol sutures unprompted.

The primary outcome measure was returns to theatre for wound complications. Baseline data showed 4 returns to theatre over a two month period $(4.40 \%$ of patients). Length of stay for each patient affected by wound ooze was also compared to the departmental mean. In the 6 month intervention period there was one return to theatre $(0.36 \%$ of patients). The observed reduction saved the department an estimated $£ 13,831$ in length of stay alone.

The standardisation of wound closure protocol, with continued reinforcement to all members of the multidisciplinary team, improves patient outcome in this group. Mobilising a group of clinicians across a variety of specialities, with one common goal, is highly effective for patients, improves multidisciplinary working and reduces cost.

\section{PROBLEM}

The Royal United Hospital, Bath (RUH) admits approximately 550 patients with neck of femur (NOF) fractures per year. The majority of these patients are treated operatively with a cemented hip hemiarthroplasty, a sliding hip screw, a long intramedullary nail or a total hip replacement. The type of intervention depends on the fracture pattern and patient factors. The operations are carried out on the emergency trauma lists by orthopaedic surgeons over a range of training grades.

This patient population is largely frail, with multiple co-morbidities, and any complications are associated with high morbidity and mortality rates. ${ }^{1}$ Return to theatre for wound ooze was targeted as a possible area for improvement opportunity within the orthopaedic department. Factors considered to be contributing to wound ooze by the team were poor closure technique, long operative times, surgeon experience and patient co-morbidity.

The aim of this project was to reduce the rate of returns to theatre for wound complications from $4.4 \%$ to $1 \%$ over a 6 month period by introducing a protocol for wound closure. A diverse team involving orthopaedic surgeons, theatre staff and orthogeriatricians was required for the project to be successful.

\section{BACKGROUND}

The incidence of NOF fractures in the United Kingdom is approximately 75,000 and is increasing each year. ${ }^{2}$ The high demand in terms of number and complexity mean the cost to the national economy is approximately $£ 2$ billion per year. ${ }^{1}$

The mortality after a hip fracture is approximately $10 \%$ at 30 days and $30 \%$ at 1 year. ${ }^{1}$ Deep wound infections are associated with $50 \%$ mortality risk and a low rate of return to pre-morbid mobility. ${ }^{1}$ Wound infection also results in increased healthcare expenditure due to length of stay, investigations and surgical costs. ${ }^{3}$ At the RUH, the mean length of stay for NOF fracture patients was 17.3 days in $2015 .^{4}$

The rate of re-operation within 30 days at the RUH Bath was $2.1 \%$ in the 2015 National Hip Fracture Database report. ${ }^{4}$ Nationally the rate of return to theatre for any reason stands at $1-2 \% .^{4}$ Reasons for re-operation included failure of fixation, wound complications and dislocation. Early mobilisation from the day following surgery is thought to reduce lengths of stay, thromboembolic disease, pressure sores and lower respiratory tract infections, however, this may place greater strain on wound healing. ${ }^{1}$ 


\section{BASELINE MEASUREMENT}

The period for baseline measurement was two months between the 1st December 2015 and the 1st February 2016. The primary outcome measure was returns to theatre for wound complications. Wound complications were defined as dehiscence, ooze, infection and haematoma. The contemporaneous database of NOF fractures was checked on the electronic theatre list for returns to theatre. This was double-checked with the NOF fracture database which records any complications and returns to theatre. The trust surgical site infection database was also cross-checked. 91 patients received operations for NOF fracture during the baseline period, 4 of whom $(4.40 \%)$ returned to theatre for wound complications.

Data collected for the 4 patients included; age, date of injury, date of surgery, type of surgery, closure method, pre and post op haemoglobin levels, transfusion rates and length of stay in an acute hospital setting. All 4 patients underwent hip hemi-arthroplasty for intracapsular NOF fractures, using a variety of closure regimen.

The mean length of stay for hip fracture patients during the baseline measurement period was 17.7 days. The mean length of stay for the patients who underwent a return to theatre was 42.0 days.

\section{DESIGN}

After discussion amongst the consultant body, a standardised wound closure protocol was proposed to reduce the $4.4 \%$ wound complication rate. A Plan-Do-Study-Act (PDSA) model for improvement addressed all aspects of protocol implementation over the 6 month intervention period. A variety of agencies including theatre nursing staff, surgical trainees, staff grade and consultant surgeons were identified. Proposed interventions included advertising the protocol around the theatre and orthopaedic departments, education sessions for all surgeons and scrubs teams, and modification of the departmental induction for rotating trainees.

Table one describes the agreed protocol for hip hemiarthroplasty closure.

Table 1 Closure protocol for hip hemi-arthroplasty

Capsule 2 absorbable monofilament (Vicryl@ Ethicon

Inc, USA) interrupted

Tendon 2 absorbable monofilament (Vicryl® Ethicon

Inc, USA) interrupted with running overlay

Fascia 2 absorbable monofilament (Vicryl ${ }^{\circledR}$ Ethicon

Inc, USA) continuous

Subcutaneous 0 absorbable monofilament (Vicryl ${ }^{\circledR}$

Ethicon Inc, USA) interrupted

Dermal 2/0 absorbable monofilament (Vicryl ${ }^{\circledR}$

Ethicon Inc, USA) continuous

Subcuticular 3/0 absorbable monofilament (Biosyn ${ }^{\mathrm{TM}}$

Medtronic, USA) continuous

Skin glue (Dermabond® Ethicon Inc, USA)

Dressing non-adherent dressing (Opsite $\diamond$ Smith \& Nephew plc)
Table two describes the agreed protocol for sliding hip screw closure.

Table 2 Closure protocol for sliding hip screw

Fascia 2 absorbable monofilament (Vicryl ${ }^{\circledR}$ Ethicon Inc, USA) continuous

Fat 0 absorbable monofilament (Vicryl ${ }^{\circledR}$ Ethicon Inc, USA) interrupted

Deep dermal 2/0 absorbable monofilament (Vicryl ${ }^{\circledR}$ Ethicon Inc, USA) continuous

Subcuticular 3/0 absorbable monofilament (Biosyn ${ }^{\mathrm{TM}}$ Medtronic, USA) continuous (no knots in wound)

Skin glue (Dermabond ${ }^{\circledR}$ Ethicon Inc, USA)

Dressing non-adherent dressing (Opsite $\diamond$ Smith \& Nephew plc)

\section{STRATEGY}

Data was collected prospectively for each patient returning to theatre for a wound complication. Operation and re-operation notes were scrutinised and method of closure and designated protocol compliant if every layer of closure was included.

The intervention during the first PDSA cycle (figure 1) was the institution, as departmental policy, of the new closure protocol developed by the lead orthopaedic consultant. This was introduced at the monthly audit meeting to those assembled and an email notification sent to all members of the department.

PDSA cycle 2 was a period of implementation and education. The education consisted of a repeat email and a teaching session to all members of the surgical team. A two week period of daily reminders in the trauma meeting was included in this cycle of change.

PDSA cycle 3 targeted sustaining the improvement through advertising the protocol and involving the multidisciplinary team to prompt surgeons in theatre. A poster was displayed in prominent locations including trauma theatres, theatre coffee room and the trauma meeting room. The posters acted as a visual prompt for surgeons and scrub staff to use the correct closure methods. The scrub teams were empowered to question deviations from the new protocol, thus engaging the multi-disciplinary team in culture change. The shift in culture was completed when scrub teams began to routinely open the protocol sutures without prompting.

Prospective data collecting was extremely encouraging however, the new rotation of trainees into the department was seen as a threat to sustainability. The baseline results, ongoing data and closure protocol was presented to the new trainees during induction. This session was reinforced with an email to the new trainees, completing PDSA cycle 4 .

\section{RESULTS}

Two-hundred and seventy-six patients with NOF fractures were admitted to the hospital during the 6 month intervention period from February 1st 2016 to August 1st 2016. One patient $(0.36 \%)$ returned to theatre for a wound 


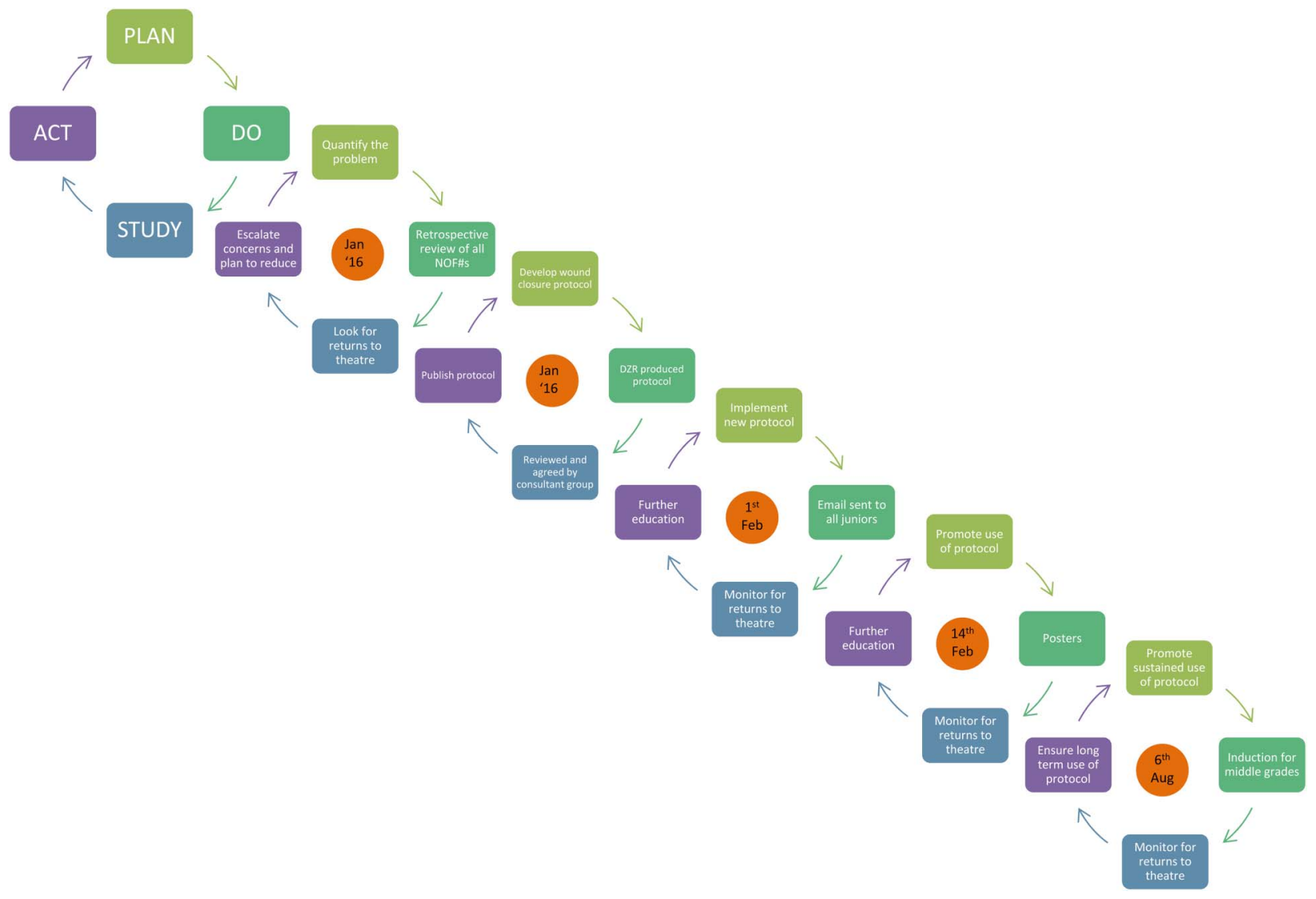

Figure 1 PDSA Cycles

complication during this time, representing a significant reduction in the primary outcome measure from $4.40 \%$. Using a Chi-squared distribution, these results indicate $p=0.018$ for the null hypothesis (that the intervention does not change rates of return to theatre for wound complications). The primary and re-operation wounds for this patient were closed according to the protocol.

Figure two shows a run chart for the 4 PDSA cycles used during the project.

The mean length of stay in the acute hospital setting was 17.7 days during the baseline phase. This reduced to 16.5 days during the intervention phase. Assuming a cost of $£ 300$ per inpatient day, this reduction in length of stay reduced costs by approximately $£ 16,200$ over the 6 months. The excess cost in terms of length of stay (mean 42 days) for the 4 wound complication patients in the two month baseline period, were $£ 30,500$. The excess cost in terms of length of stay (42 days) for the only patient to return to theatre during the intervention period was $£ 7,677$. This is an absolute saving of $£$ $£ 22,822$ for returns to theatre for wound complications and corrected for the number of months, constitutes a monthly saving of $£ 13,831$ post-improvement.

\section{LESSONS AND LIMITATIONS}

Lessons: The standardisation of wound closure protocol, with continued reinforcement to all members of the multidisciplinary team, reduces wound complications, length of stay, returns to theatre and cost. Mobilising a group of clinicians across a variety of specialities, with one common goal, is highly effective in terms of a reduction in adverse events and improves multidisciplinary working.

Challenges: Achieving a change in practice across all grades of surgeon was challenging as existing practices were well embedded. Due to training requirements, a regular turnover of staff threatened sustainability and measures to tackle this were built in to the improvement strategy.

Sustainable: Sustainability has been achieved by updating the induction programmes for the junior and senior trainees. The use of the data collected in this project is a powerful adjunct to this induction programme and the sustained efforts to continue collecting surgical site infection data by the department, as a standard of care, have seen sustained improvement. In the current cohort of trainees there have been no returns to theatre for wound complications for the 77 NOF fracture patients. Whilst trainees rotate, theatre staff are relatively more static, as are the consultant body. With the involvement of these two groups, as sustained culture change has been achieved.

Limitations: return to theatre is an extreme outcome for wound complications and there may be many other patients who have some level of wound complication not 


\section{$\%$ return to theatre}

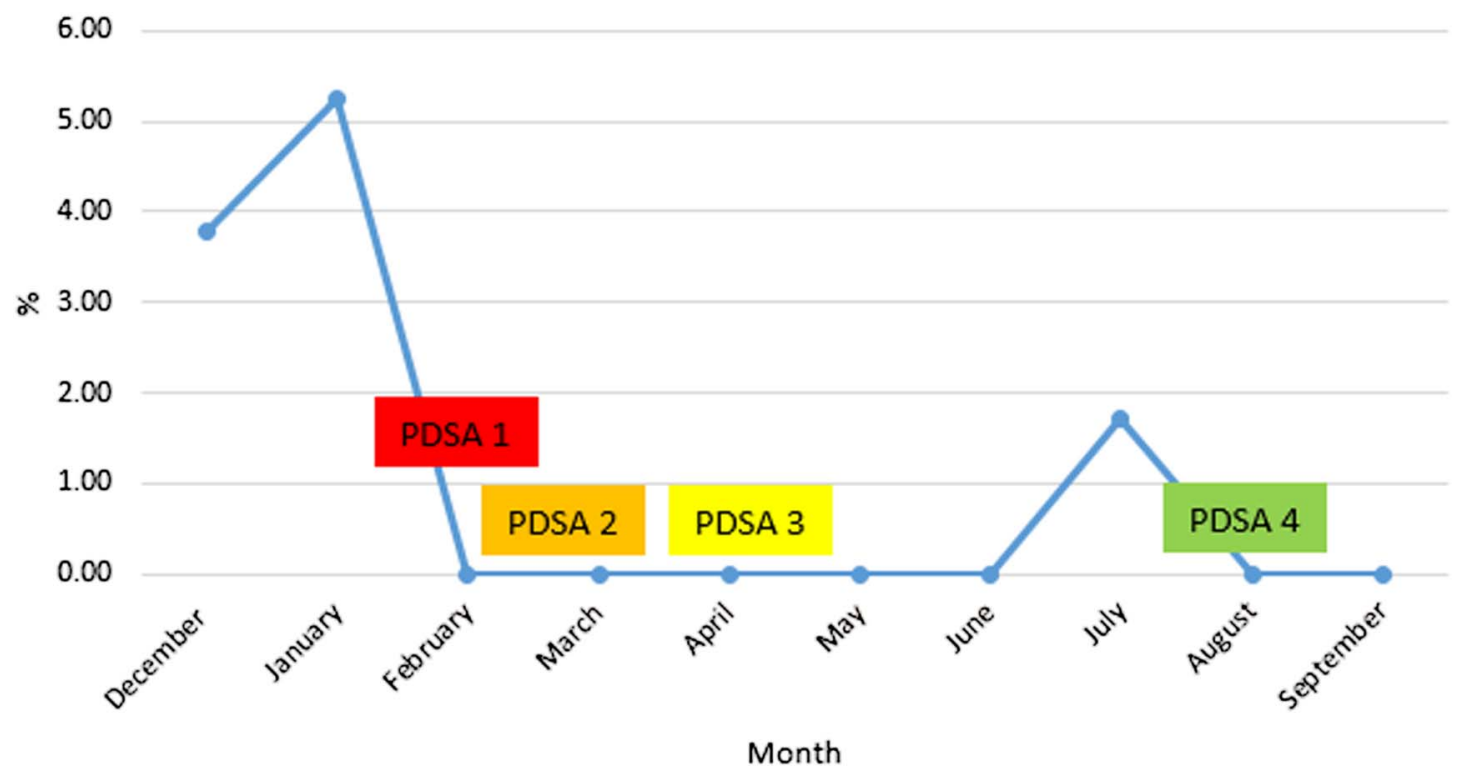

Figure 2 Run chart

severe enough to warrant surgical intervention. Quantifying wound ooze is difficult to achieve objectively, however rates of surgical site infections (SSIs) and returns to theatre could be compared.

Cost effective: The costs of this study were minimal and achieved during non-paid hours. The reduction in costs from length of stay only were $£ 13,831$. This is offset by a possible increase in suture and glue materials although it was not possible to calculate these costs across the entire cohort.

\section{CONCLUSION}

The new wound closure protocol significantly reduced the number of hip fracture patients requiring a return to theatre for wound complications and reduced costs. This protocol is easily transferable to other hospital trusts. By adopting similar multidisciplinary involvement and a clinician induction programme, sustained improvements are achievable, sustainable and reproducible.

Acknowledgements Mr Derek Robinson - supervising consultant
Declaration of interests Nothing to declare.

Ethical approval This project was submitted to the local research, audit and QIP department at the RUH Bath. No further ethical review was required from them.

Open Access This is an open-access article distributed under the terms of the Creative Commons Attribution Non-commercial License, which permits use, distribution, and reproduction in any medium, provided the original work is properly cited, the use is non commercial and is otherwise in compliance with the license. See:

- http://creativecommons.org/licenses/by-nc/2.0/

- http://creativecommons.org/licenses/by-nc/2.0/legalcode

\section{REFERENCES}

1. NICE, 2011 (updated 2014). CG124 - Hip fracture: The management of hip fracture in adults. Available at: https://www.nice.org.uk/ guidance/cg124/ (accessed 7 August 2016).

2. British Orthopaedic Association. BOA/BGS Blue Book - The Care of Patients with Fragility Fractures (Guideline Ref ID: BOA2007)

3. Edwards C, Counsell A, Boulton C, Moran CG. Early infection after hip fracture surgery - risk factors, costs and outcome. J Bone Joint Surg [Br]. 2008; (90-B):770-7.

4. National Hip Fracture Database. Available at http://www.nhfd.co.uk/ (accessed 23 August 2016). 